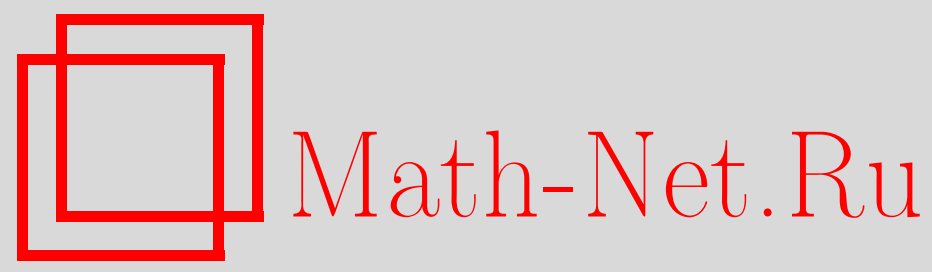

М. Р. Достанич, Норма и регуляризированный след преобразования Коши, Матем. заметки, 2005, том 77, выпуск 6, 844-853

DOI: https://doi.org/10.4213/mzm2539

Использование Общероссийского математического портала Math-Net.Ru подразумевает, что вы прочитали и согласны с пользовательским соглашением http://www.mathnet.ru/rus/agreement

Параметры загрузки:

IP : 52.6 .47 .48

26 апреля 2023 г., $07: 16: 12$

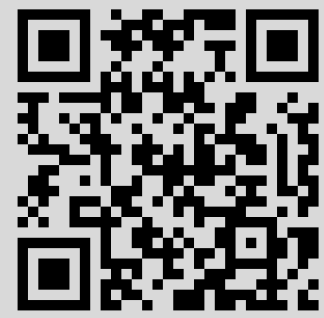




\title{
НОРМА И РЕГУЛЯРИЗИРОВАННЫЙ СЛЕД ПРЕОБРАЗОВАНИЯ КОШИ
}

\author{
М.Р. Достанич
}

В статье получена норма преобразования Коши $C$ на пространстве $L^{2}(D, d \mu)$, где $d \mu=\omega(|z|) d A(z)$. Также (в случае $\omega \equiv 1$ ) получен первый регуляризированный след оператора $C^{*} C$ на пространстве $L^{2}(\Omega)$. Эти результаты иллюстрируются различными примерами, в которых функция $\omega$ и область $\Omega$ выбираются некоторьм специальным образом.

Библиограбоия: 12 названий.

1. Введение. Пусть $D=\{z:|z|<1\}$ и $\omega \in C^{1}(0,1)$ - некоторая положительная функция такая, что

$$
\frac{\omega(x)}{x} \int_{0}^{x} t \omega(t) d t \in L^{1}(0,1) .
$$

Пусть $d \mu=\omega(|z|) d A(z)$, где $d A$ - мера Лебега. Через $L^{2}(D, d \mu)$ обозначим пространство Лебега с мерой $d \mu$ на единичном круге $D$. Пусть $C_{\omega}: L^{2}(D, d \mu) \rightarrow L^{2}(D, d \mu)-$ оператор, задаваемьй соотношением

$$
C_{\omega} f(z)=-\frac{1}{\pi} \int_{D} \frac{f(\xi)}{\xi-z} d \mu(\xi)
$$

(т.е. интегральный оператор Коши или преобразование Коши).

В данной статье определяется норма оператора $C_{\omega}$ (через функцию $\omega$, характеризующую граничную задачу). В случае $\omega \equiv 1$ эта задача была решена в работе [1], где было показано, что норма соответствующего оператора равна $2 / j_{0}$; здесь $j_{0}$ - наименьший положительньй нуль функции Бесселя $J_{0}$.

Во второй части работы, рассматривается оператор

$$
C: L^{2}(\Omega) \rightarrow L^{2}(\Omega), \quad C f(z)=-\frac{1}{\pi} \int_{\Omega} \frac{f(\xi)}{\xi-z} d A(\xi)
$$

где $\Omega$ - некоторая ограниченная односвязная область в $\mathbb{C}$. При определенных условиях находится регуляризированный след оператора $C^{*} C$ (который не принадлежит классу следов). Более подробное описание сингулярных значений компактных операторов, а также идеалов Шаттена $c_{p}$, можно найти в [2] (через $c_{1}$ мы обозначаем класс следов). 


\section{2. Основные результаты.}

Teopema 1. Пусть $\omega \in C^{1}(0,1), \omega(x)>0$ на $(0,1) u$

$$
\frac{\omega(x)}{x} \int_{0}^{x} t \omega(t) d t \in L^{1}(0,1) .
$$

Тогда $\left\|C_{\omega}\right\|=2 \sqrt{\lambda^{*}}$, где $\lambda^{*}-$ наибольшее значение $\lambda$ такое, что граничная задача

$$
\begin{gathered}
\lambda \frac{d}{d x}\left(\frac{y^{\prime}}{x \omega(x)}\right)+y \frac{\omega(x)}{x}=0, \\
y\left(0_{+}\right)=0, \quad \lim _{x \rightarrow 1-0} \frac{y^{\prime}(x)}{\omega(x)}=0
\end{gathered}
$$

имеет решение $y \in C^{2}(0,1)$, удовлетворяющее условиям $y(x)>0$ и $y^{\prime}(x)>0$ на $(0,1)$.

ЗАмечание 1. Функция $\omega(x)=x^{\alpha}, \alpha>-1$, удовлетворяет условиям теоремы 1 . Следовательно, для определения нормы соответствующего оператора $C_{\omega}$, нужно найти наибольшее значение $\lambda$, при котором граничная задача

$$
\left\{\begin{array}{l}
y^{\prime \prime} x^{2}-(\alpha+1) x y^{\prime}+y \cdot \frac{x^{(2 \alpha+2)}}{\lambda}=0 \\
y(0+)=0, \quad y^{\prime}(1-0)=0
\end{array}\right.
$$

имеет решение $y \in C^{2}(0,1)$, удовлетворяюшее условиям $y(x)>0$ и $y^{\prime}(x)>0$ при $x \in(0,1)$.

Решая дифференциальное уравнение (2) (см. [3]), получим

$$
y(x)=x^{(\alpha+2) / 2} \cdot Z_{\nu}\left(\frac{x^{\alpha+1}}{(\alpha+1) \sqrt{\lambda}}\right), \quad \nu=\frac{\alpha+2}{2 \alpha+2},
$$

где $Z_{\nu}=c_{1} J_{\nu}+c_{2} Y_{\nu}\left(J_{\nu}\right.$ и $Y_{\nu}-$ функции Бесселя первого и второго рода).

Так как $y(0+)=0$, из поведения функции $Y_{\nu}, \nu=(\alpha+2) /(2 \alpha+2)$, в окрестности точки $x=0$ следует, что $c_{2}=0$.

Отсюда следует, что

$$
y(x)=x^{(\alpha+2) / 2} J_{\nu}\left(\frac{x^{\alpha+1}}{(\alpha+1) \sqrt{\lambda}}\right)
$$

(можно положить $c_{1}=1$ ). Так как

$$
J_{\nu}^{\prime}(z)+\frac{\nu}{z} J_{\nu}(z)=J_{\nu-1}(z)
$$

полагая, что $z=x^{\alpha+1} /((\alpha+1) \sqrt{\lambda})$, получим

$$
y^{\prime}(x)=\frac{1}{\sqrt{\lambda}} x^{(3 \alpha+2) / 2} J_{\nu-1}\left(\frac{x^{\alpha+1}}{(\alpha+1) \sqrt{\lambda}}\right) .
$$

Из условия $y^{\prime}(1-0)=0$ следует, что

$$
J_{\nu-1}\left(\frac{1}{(\alpha+1) \sqrt{\lambda}}\right)=0 .
$$


Пусть $j_{\alpha}-$ наименьший положительный нуль функции $J_{\nu-1}$ (т.е. функции $J_{-\alpha /(2 \alpha+2)}$, так как $\nu=(\alpha+2) /(2 \alpha+2))$.

Тогда, очевидно, $y^{\prime}(x)>0$ на отрезке $(0,1)$. Из того, что наименьший положительный нуль функции $J_{\nu-1}$ меньше, чем наименьший положительный нуль функции $J_{\nu}$ (см. $[4$, с. 70$])$, следует, что $y(x)>0$ на отрезке $(0,1)$. Таким образом, наибольшее число $\lambda^{*}$ (при котором задача (2) имеет решение $y \in C^{2}(0,1)$ такое, что $y(x)>0$ и $y^{\prime}(x)>0$ на $(0,1))$ удовлетворяет условию

$$
\frac{1}{(\alpha+1) \sqrt{\lambda^{*}}}=j_{\alpha}
$$

Из этого условия и из теоремы 1 следует, что при $\omega(x)=x^{\alpha}, \alpha>-1$, имеет место равенство

$$
\left\|C_{\omega}\right\|=\frac{2}{(\alpha+1) j_{\alpha}} .
$$

Случай $\alpha=0$ рассматривался в [1].

3. Доказательство теоремы 1. Для доказательства теоремы нам понадобится ряд лемм.

Пусть $A_{m}, m \in \mathbb{Z},-$ операторы на пространстве $L^{2}(0,1)$, определенные следующим образом:

$$
A_{m} f(r)= \begin{cases}2 \int_{0}^{r} \sqrt{\omega(r) \omega(t)}\left(\frac{t}{r}\right)^{1 / 2-m} f(t) d t, & m \leqslant 0 \\ 2 \int_{1}^{r} \sqrt{\omega(r) \omega(t)}\left(\frac{r}{t}\right)^{m-1 / 2} f(t) d t, & m \geqslant 1 .\end{cases}
$$

Лемма 1. Пусть $K_{0}$ - минимальное значение константы $K$ такой, что неравенство

$$
\int_{0}^{1} \int_{0}^{2 \pi}\left|\sum_{m} e^{i m \theta}\left(A_{m} \Phi_{m}\right)(r)\right|^{2} d r d \theta \leqslant K^{2} \int_{0}^{1} \int_{0}^{2 \pi}\left|\sum_{m} e^{i m \theta} \Phi_{m}(r)\right|^{2} d r d \theta
$$

выполняется при любых (конечных) значениях функиии $\Phi_{m} \in L^{2}(0,1)$. Тогда $\left\|C_{\omega}\right\|=K_{0} .($ Здесь $r \in(0,1), \theta \in(0,2 \pi)$.)

ДокАЗАТЕЛЬСТво. Пусть $z=r e^{i \theta}$. Тогда система функций

$$
\left\{f_{m n}\right\}_{m \in \mathbb{Z}, n \in \mathbb{N}}, \quad \text { где } f_{m n}(z)=e^{i m \theta} J_{m}\left(j_{m n} r\right),
$$

полна в пространстве $L^{2}(D, d \mu)[5]$. Здесь $\left\{j_{m n}\right\}_{n=1}^{\infty}-$ множество положительных нулей функции Бесселя $J_{m}, m \in \mathbb{Z}$. Непосредственно вычисляется, что

$$
C_{\omega} f_{m n}(z)=\left\{\begin{array}{cll}
2 z^{m-1} \int_{1}^{|z|} r^{1-m} J_{m}\left(j_{m n} r\right) \omega(r) d r, & m \geqslant 1, & n \in \mathbb{N} \\
2 z^{m-1} \int_{0}^{|z|} r^{1-m} J_{m}\left(j_{m n} r\right) \omega(r) d r, & m \leqslant 0, & n \in \mathbb{N}
\end{array}\right.
$$

Предположим, что неравенство (3) выполняется при любом выборе функций $\Phi_{m} \in$ $L^{2}(0,1)$. Полагая, что $\Phi_{m}$ являются функциями вида

$$
r \mapsto \sum_{n} c_{m n} \sqrt{r \omega(r)} J_{m}\left(j_{m n} r\right)
$$


где сумма конечна, применяя (4) и принимая во внимание определение операторов $A_{m}$, мы видим, что неравенство (3) можно записать в виде

$$
\int_{D}\left|C_{\omega}\left(\sum_{m, n} c_{m n} f_{m n}\right)\right|^{2} d \mu \leqslant K^{2} \int_{D}\left|\sum_{m, n} c_{m n} f_{m n}\right|^{2} d \mu
$$

Так как система функций $\left\{f_{m n}\right\}_{m \in \mathbb{Z}, n \in \mathbb{N}}$ полна в $L^{2}(D, d \mu)$, из предыдущего неравенства следует, что

$$
\int_{D}\left|C_{\omega} f\right|^{2} d \mu \leqslant K^{2} \int_{D}|f|^{2} d \mu \quad \forall f \in L^{2}(D, d \mu)
$$

т.е. $\left\|C_{\omega}\right\| \leqslant K$; таким образом, получаем, что

$$
\left\|C_{\omega}\right\| \leqslant K_{0}
$$

Обратно, из неравенства

$$
\left\|C_{\omega}\left(\sum_{m, n} c_{m n} f_{m n}\right)\right\|^{2} \leqslant\left\|C_{\omega}\right\|^{2}\left\|\sum_{m, n} c_{m n} f_{m n}\right\|^{2}
$$

где $\|\varphi\|^{2}=\int_{D}|\varphi|^{2} d \mu$, следует, что

$$
\int_{0}^{1} \int_{0}^{2 \pi}\left|\sum_{m} e^{i m \theta} A_{m} \Phi_{m}(r)\right|^{2} d r d \theta \leqslant\left\|C_{\omega}\right\|^{2} \int_{0}^{1} \int_{0}^{2 \pi}\left|\sum_{m} e^{i m \theta} \Phi_{m}(r)\right|^{2} d r d \theta
$$

где

$$
\Phi_{m}(r)=\sum_{n} c_{m n}(r \omega(r))^{1 / 2} J_{m}\left(j_{m n} r\right) .
$$

Так как система функций $\left\{(r \omega(r))^{1 / 2} J_{m}\left(j_{m n} r\right)\right\}_{n=1}^{\infty}$ полна в пространстве $L^{2}(0,1)$ при любом $m \in \mathbb{Z}$, то неравенство (5) также выполняется для любой произвольной функции $\Phi_{m} \in(0,1)$ (так как все операторы $A_{m}$ ограничены на $\left.L^{2}(0,1)\right)$. Отсюда следует неравенство

$$
K_{0}^{2} \leqslant\left\|C_{\omega}\right\|^{2}
$$

которое завершает доказательство леммы.

ЛЕмма 2. Пусть $T: L^{2}(0,1) \rightarrow L^{2}(0,1)$ - оператор, определенный как

$$
T f(x)=\int_{0}^{x} \sqrt{\omega(x) \omega(y)} \sqrt{\frac{y}{x}} f(y) d y
$$

Tогда

$$
\left\|C_{\omega}\right\|=2\|T\|
$$


ДоКАЗАТЕЛЬСТво. Применяя равенство Парсеваля, из леммы 1 получаем

$$
\left\|C_{\omega}\right\|=\sup _{m \in \mathbb{Z}}\left\|A_{m}\right\| .
$$

Покажем, что

$$
\begin{aligned}
& \left\|A_{m}\right\| \leqslant\left\|A_{0}\right\|, \quad \text { если } m \leqslant 0, \\
& \left\|A_{m}\right\| \leqslant\left\|A_{1}\right\|, \quad \text { если } m \geqslant 1 .
\end{aligned}
$$

Пусть

$$
\theta(s)= \begin{cases}1, & s \geqslant 0 \\ 0, & s<0\end{cases}
$$

а также пусть $m \leqslant 0$ и $f, g \in L^{2}(0,1)$. Тогда имеем

$$
\left(A_{m} f, g\right)=2 \int_{0}^{1} \int_{0}^{1} \overline{g(x)} \theta(y-x) \sqrt{\omega(x) \omega(y)}\left(\frac{y}{x}\right)^{1 / 2-m} f(y) d x d y .
$$

Следовательно, принимая во внимание неравенство

$$
\theta(y-x)\left(\frac{y}{x}\right)^{1 / 2-m} \leqslant \theta(y-x) \sqrt{\frac{y}{x}}
$$

получаем

$$
\begin{aligned}
\left|\left(A_{m} f, g\right)\right| & \leqslant 2 \int_{0}^{1} \int_{0}^{1}|g(x)||f(y)| \sqrt{\omega(x) \omega(y)} \sqrt{\frac{y}{x}} d x d y \\
& =\left|\left(A_{0}|f|,|g|\right)\right| \leqslant\left\|A_{0}\right\| \cdot\|f\| \cdot\|g\|,
\end{aligned}
$$

т.e.

$$
\left\|A_{m}\right\| \leqslant\left\|A_{0}\right\| .
$$

Неравенство (7) доказывается аналогично. Таким образом,

$$
\left\|C_{\omega}\right\|=\max \left\{\left\|A_{0}\right\|,\left\|A_{1}\right\|\right\} .
$$

Из равенства $A_{1}^{*}=-A_{0}$ следует, что

$$
\left\|C_{\omega}\right\|=\left\|A_{0}\right\|=2\|T\|
$$

Докажем теперь теорему 1.

Из леммы 2 следует, что достаточно определить норму оператора $T$. Эта норма равна $\sqrt{\lambda^{*}}$, где $\lambda^{*}$ - наименьшее значение константы $\lambda$, при котором неравенство

$$
\int_{0}^{1} \frac{\omega(x)}{x}\left|\int_{0}^{x} \sqrt{y \omega(y)} f(y) d y\right|^{2} d x \leqslant \lambda \int_{0}^{1}|f|^{2} d x
$$

выполняется при любых $f \in L^{2}(0,1)$. Если положить $g(y)=f(y) \sqrt{y \omega(y)}, W(x)=$ $\omega(x) / x$ и $M(x)=1 /(x \omega(x))$, то предыдущее неравенство примет вид

$$
\int_{0}^{1} W(x)\left|\int_{0}^{x} g(y) d y\right|^{2} d x \leqslant \lambda \int_{0}^{1}|g(x)|^{2} M(x) d x, \quad g \in L^{2}((0,1), M d x) .
$$


Отметим, что оператор $T_{1} f(x)=\omega(x) \int_{0}^{x} f(t) d t$ является компактным на $L^{2}((0,1)$, $M d x)$. (Кроме того, в силу условия (1), этот оператор является оператором Гильберта-Шмидта.)

Применим теорему 1 из работы [6] в случае, когда $p=r=2, q=0, W(x)=\omega(x) / x$, $M(x)=1 /(x \omega(x))$. Видно, что наименьшее значение $\lambda$, при котором (8) выполняется при любом $g \in L^{2}((0,1), M d x)$, равно наибольшему значению $\lambda$, при котором граничная задача

$$
\begin{gathered}
\lambda \frac{d}{d x}\left(\frac{y^{\prime}}{x \omega(x)}\right)+y \frac{\omega(x)}{x}=0, \\
y\left(0_{+}\right)=0, \quad \lim _{x \rightarrow 1-0} \frac{y^{\prime}(x)}{\omega(x)}=0,
\end{gathered}
$$

имеет решение $y \in C^{2}(0,1)$, удовлетворяющее условиям $y(x)>0$ и $y^{\prime}(x)>0$ при $x \in(0,1)$.

Отсюда следует утверждение теоремы.

4. Регуляризированный след преобразования Коши. Пусть $\Omega$ - ограниченная односвязная область с аналитической границей в пространстве $\mathbb{C}$. Известно [7], что преобразование Коши $C: L^{2}(\Omega) \rightarrow L^{2}(\Omega)$,

$$
C f(z)=-\frac{1}{\pi} \int_{\Omega} \frac{f(\xi)}{\xi-z} d A(\xi),
$$

имеет следующую асимптотику сингулярных значений:

$$
s_{n}(C) \sim \sqrt{\frac{|\Omega|}{\pi n}}, \quad n \rightarrow \infty
$$

где $|\Omega|$ - плошадь области $\Omega$. Таким образом, мы видим, что оператор $C^{*} C$ не принадлежит классу $c_{1}$.

Теперь найдем сумму

$$
\sum_{n=1}^{\infty}\left(s_{n}^{2}(C)-\mu_{n}\right)
$$

где $\mu_{n}$ - некоторая специальным образом выбранная последовательность (либо некоторая конкретная последовательность, либо последовательность, связанная со спектром какого-либо хорошо известного оператора).

Предположим, что $F$ - конформное отображение области $\Omega$ на единичный круг $D(F$ можно аналитически продолжить на некоторую окрестность $\bar{\Omega}$ так, чтобы $F^{\prime}(z) \neq 0$ на $\bar{\Omega})$.

Через $\left\{\lambda_{n}\right\}_{n=1}^{\infty}, 0<\lambda_{1}<\lambda_{2} \leqslant \lambda_{3} \leqslant \cdots$, обозначим собственные значения, а через $\left\{u_{n}\right\}_{n=1}^{\infty}-$ ортонормированную систему (в $L^{2}(\Omega)$ ) соответствуюших собственных функций лапласиана Дирихле, т.е. граничной задачи

$$
-\Delta u=\lambda u,\left.\quad u\right|_{\partial \Omega}=0 .
$$

Отметим, что $\left\{u_{n}\right\}_{n=1}^{\infty}$ является ортонормированным базисом в пространстве $L^{2}(\Omega)$. 
Теорема 2. Для любого $n \in \mathbb{N}$ выполняется неравенство $s_{n}(C) \geqslant 2 / \sqrt{\lambda_{n}}$, сходится ряд $\sum_{n=1}^{\infty}\left(s_{n}^{2}(C)-4 / \lambda_{n}\right)$ и имеет место равенство

$$
\sum_{n=1}^{\infty}\left(s_{n}^{2}(C)-\frac{4}{\lambda_{n}}\right)=\frac{1}{\pi^{2}} \int_{\Omega} \int_{\Omega}\left|\frac{1}{z-\xi}-\frac{F^{\prime}(z)}{F(z)-F(\xi)}-\frac{\overline{F(\xi)} F^{\prime}(z)}{1-\overline{F(\xi)} F(z)}\right|^{2} d A(\xi) d A(z)
$$

ЗАмЕчаниЕ 2 . В случае $\Omega=D$ все сингулярные значения преобразования Коши были определены в работе [12]. Все они двукратные и равны $\left\{2 / j_{m n}\right\}, m=0,1,2, \ldots$, $n=1,2, \ldots$, где $\left\{j_{m n}\right\}_{n=1}^{\infty}-$ положительные нули функции Бесселя $J_{m}$.

Однако спектр лапласиана Дирихле для единичного круга содержит двукратные собственные значения $\left\{j_{m n}^{2}\right\}, m, n \in \mathbb{N}$, так же, как и однократные собственные значения $\left\{j_{0 n}^{2}\right\}_{n=1}^{\infty}$. Применим (9) в случае $\Omega=D$. Тогда мы можем положить $F(z)=z$ и, таким образом, получаем

$$
4 \sum_{n=1}^{\infty} \frac{1}{j_{0 n}^{2}}=\frac{1}{\pi} \int_{D} \int_{D}\left|\frac{\bar{\xi}}{1-z \bar{\xi}}\right|^{2} d A(\xi) d A(z)
$$

Легко видеть, что правая часть равна единице и, следовательно,

$$
\sum_{n=1}^{\infty} \frac{1}{j_{0 n}^{2}}=\frac{1}{4}
$$

Тот же результат был получен в работе [4, с. 72-73] с помощью теоремы Адамара о факторизации.

5. Доказательство теоремы 2. Функция Грина лапласиана Дирихле для области $\Omega$ имеет вид

$$
-\frac{1}{2 \pi} \ln \left|\frac{F(z)-F(\xi)}{1-\overline{F(\xi)} F(z)}\right|
$$

и, таким образом, для любых $n \in \mathbb{N}$ мы имеем

$$
\int_{\Omega}\left(-\frac{1}{2 \pi} \ln \left|\frac{F(z)-F(\xi)}{1-\overline{F(\xi)} F(z)}\right|\right) u_{n}(\xi) d A(\xi)=\frac{1}{\lambda_{n}} u_{n}(z) .
$$

Дифференцируя обе части по $z$, получаем

$$
\int_{\Omega} \frac{1}{\pi}\left[\frac{F^{\prime}(z)}{F(z)-F(\xi)}+\frac{\overline{F(\xi)} F^{\prime}(z)}{1-\overline{F(\xi)} F(z)}\right] u_{n}(\xi) d A(\xi)=-\frac{2}{\sqrt{\lambda_{n}}} \theta_{n}(z)
$$

где

$$
\theta_{n}(z)=\frac{2}{\sqrt{\lambda_{n}}} \frac{\partial u_{n}}{\partial z}
$$

Отсюда следует, что $\theta_{n} \in C(\bar{\Omega})$ (см. [8, с. 39-41]), а из формулы Коши-Грина [8] следует, что

$$
\int_{\Omega} \theta_{n}(z) \overline{\theta_{m}(z)} d A(z)=\delta_{n m}
$$


Пусть $A, B: L^{2}(\Omega) \rightarrow L^{2}(\Omega)$ - операторы, определенные равенствами

$$
\begin{aligned}
& A f(z)=\int_{\Omega} \frac{1}{\pi}\left[\frac{F^{\prime}(z)}{F(z)-F(\xi)}+\frac{\overline{F(\xi)} F^{\prime}(z)}{1-\overline{F(\xi)} F(z)}\right] f(\xi) d A(\xi) \\
& B f(z)=\int_{\Omega} \frac{1}{\pi}\left[\frac{1}{z-\xi}-\frac{F^{\prime}(z)}{F(z)-F(\xi)}-\frac{\overline{F(\xi)} F^{\prime}(z)}{1-\overline{F(\xi)} F(z)}\right] f(\xi) d A(\xi) .
\end{aligned}
$$

Следовательно, $A u_{n}=-\left(2 / \sqrt{\lambda_{n}}\right) \theta_{n}$ и, таким образом, мы получаем

$$
A f=\sum_{n=1}^{\infty}-\frac{2}{\sqrt{\lambda_{n}}}\left(f, u_{n}\right) \theta_{n}
$$

$\left(\right.$ здесь $(\cdot, \cdot)$ - скалярное произведение в пространстве $\left.L^{2}(\Omega)\right)$, откуда следует, что

$$
s_{n}(A)=\frac{2}{\sqrt{\lambda_{n}}} .
$$

Для компактного оператора $S$ через $\lambda_{n}(S)$ и $s_{n}(S)$ обозначим соответственно его $n$-е собственное значение и его $n$-е сингулярное значение.

Из леммы 7 в работе [9], теоремы Ки-Фана [2] и теоремы Бирмана-Соломяка (о росте сингулярных значений интегральных операторов с гладкими ядрами) следует, что

$$
s_{n}(B) \sim \operatorname{const} \frac{1}{n^{2}}, \quad n \rightarrow \infty
$$

т.е.

$$
\lambda_{n}\left(B^{*} B\right) \sim \operatorname{const} \frac{1}{n^{2}}, \quad n \rightarrow \infty
$$

Покажем, что

$$
A^{*} B=B^{*} A=0 .
$$

Так как $\left\{u_{n}\right\}_{n=1}^{\infty}$ является ортонормированным базисом в $L^{2}(\Omega)$, то достаточно показать, что для любых $m, n \in \mathbb{N}$ вьполняется равенство

$$
\left(A u_{n}, B u_{m}\right)=0 .
$$

Следовательно,

$$
\begin{aligned}
& \left(B u_{m}, A u_{n}\right)=\left(B u_{m},-\frac{2}{\sqrt{\lambda_{n}}} \theta_{n}\right) \\
& \quad=-\frac{2}{\sqrt{\lambda_{n}}} \int_{\Omega} \overline{\theta_{n}}(z) d A(z) \int_{\Omega} \frac{1}{\pi}\left[\frac{1}{z-\xi}-\frac{F^{\prime}(z)}{F(z)-F(\xi)}-\frac{\overline{F(\xi)} F^{\prime}(z)}{1-\overline{F(\xi)} F(z)}\right] u_{m}(\xi) d A(\xi)
\end{aligned}
$$

(теорема Фубини)$$
=-\frac{2}{\sqrt{\lambda_{n}}} \int_{\Omega} u_{n}(\xi) d A(\xi) \int_{\Omega} \frac{1}{\pi}\left[\frac{1}{z-\xi}-\frac{F^{\prime}(z)}{F(z)-F(\xi)}-\frac{\overline{F(\xi)} F^{\prime}(z)}{1-\overline{F(\xi)} F(z)}\right] \overline{\theta_{n}}(z) d A(z)
$$$$
=-\frac{4}{\pi \lambda_{n}} \int_{\Omega} u_{n}(\xi) d A(\xi) \int_{\Omega} \frac{\partial}{\partial \bar{z}}\left[\overline{u_{n}(z)}\left(\frac{1}{z-\xi}-\frac{F^{\prime}(z)}{F(z)-F(\xi)}-\frac{\overline{F(\xi)} F^{\prime}(z)}{1-\overline{F(\xi)} F(z)}\right) d A(z)\right]
$$

(формула Коши-Грина)

$$
\begin{aligned}
& =-\frac{4}{\pi \lambda_{n}} \int_{\Omega} u_{n}(\xi) d A(\xi)\left[\frac{1}{2 i} \int_{\partial \Omega} \overline{u_{n}(z)}\left(\frac{1}{z-\xi}-\frac{F^{\prime}(z)}{F(z)-F(\xi)}-\frac{\overline{F(\xi)} F^{\prime}(z)}{1-\overline{F(\xi)} F(z)}\right) d z\right] \\
& =0
\end{aligned}
$$


потому что $\left.u_{n}\right|_{\partial \Omega}=0$. Так как $C=A+B$, то из (11) следует, что

$$
C^{*} C=A^{*} A+B^{*} B
$$

откуда вытекает, что $C^{*} C \geqslant A^{*} A$. Таким образом, применяя неравенство Гейнца, мы получаем неравенство

$$
\sqrt{C^{*} C} \geqslant \sqrt{A^{*} A}
$$

откуда следует, что $s_{n}(C) \geqslant 2 / \sqrt{\lambda_{n}}$.

Для доказательства формулы следа нам потребуется следующая лемма.

Лемма 3 [10]. Если Е и F- положительные компактные операторы на некотором комплексном аильбертовом пространстве $\mathscr{H}$ таком, что $|E-F|^{\operatorname{Re} z} \in c_{1}$, $0<\operatorname{Re} z<1$, mo $E^{z}-F^{z} \in c_{1} u$

$$
\left|E^{z}-F^{z}\right|_{1} \leqslant \frac{|\sin \pi z|}{\sin \pi(\operatorname{Re} z)}|| E-\left.\left.F\right|^{\operatorname{Re} z}\right|_{1} .
$$

(Здесь $c_{1}$ означает класс следов, символ $|S|$ обозначает $\sqrt{S^{*} S}$ и $|S|_{1}$ обозначает норму операторов $S$ в классе следов).

Из (10) и (12) следует, что при любых $k \in \mathbb{N}$ выполняется равенство

$$
s_{n}\left(\left(C^{*} C\right)^{k}-\left(A^{*} A\right)^{k}\right)=O\left(\frac{1}{n^{k+1}}\right),
$$

и, таким образом, по лемме 3 функция

$$
z \mapsto \operatorname{tr}\left(\left(C^{*} C\right)^{z}-\left(A^{*} A\right)^{z}\right)=\operatorname{tr}\left(\left(\left(C^{*} C\right)^{k}\right)^{z / k}-\left(\left(A^{*} A\right)^{k}\right)^{z / k}\right)
$$

является аналитической при $1 /(k+1)<\operatorname{Re}(z / k)<1$, т.е. при

$$
\frac{k}{k+1}<\operatorname{Re} z<k \text {. }
$$

Следовательно, функция $z \mapsto \operatorname{tr}\left(\left(C^{*} C\right)^{z}-\left(A^{*} A\right)^{z}\right)$ является аналитической при $z$ таких, что $\operatorname{Re} z>1 / 2$.

Так как $\lambda_{n}\left(A^{*} A\right)=4 / \lambda_{n}$ и асимптотика последовательности $\lambda_{n}$ известна (формула Вейля), мы имеем

$$
\lambda_{n}\left(A^{*} A\right)=\frac{|\Omega|}{\pi n}+O\left(\frac{1}{n^{3 / 2}}\right) .
$$

Отсюда и из (12) в соответствии с теоремой 6.3 в работе [11, с. 48] следует, что

$$
\lambda_{n}\left(C^{*} C\right)=\frac{|\Omega|}{\pi n}+O\left(\frac{1}{n^{4 / 3}}\right) .
$$

Рассмотрим функцию

$$
\Psi(z)=\sum_{n=1}^{\infty}\left(\lambda_{n}^{z}\left(C^{*} C\right)-\lambda_{n}^{z}\left(A^{*} A\right)\right) \quad\left(=\sum_{n=1}^{\infty}\left(\lambda_{n}^{z}\left(C^{*} C\right)-\left(\frac{4}{\lambda_{n}}\right)^{z}\right)\right) .
$$


Из упомянутой вьше асимптотики последовательностей $\lambda_{n}\left(A^{*} A\right)$ и $\lambda_{n}\left(C^{*} C\right)$ следует, что $\Psi$ является аналитической функцией при $z$ таких, что $\operatorname{Re} z \geqslant 2 / 3$.

Если $\operatorname{Re} z>1$, то оба оператора $\left(C^{*} C\right)^{z}$ и $\left(A^{*} A\right)^{z}$ принадлежат классу $c_{1}$ и, таким образом,

$$
\operatorname{tr}\left(\left(C^{*} C\right)^{z}-\left(A^{*} A\right)^{z}\right)=\operatorname{tr}\left(\left(C^{*} C\right)^{z}\right)-\operatorname{tr}\left(\left(A^{*} A\right)^{z}\right)=\Psi(z) .
$$

Отсюда в силу теоремы единственности следует, что

$$
\Psi(z) \equiv \operatorname{tr}\left(\left(C^{*} C\right)^{z}-\left(A^{*} A\right)^{z}\right)
$$

при $z$ таких, что $\operatorname{Re} z>2 / 3$. Следовательно, для любых $k \in \mathbb{N}$, имеем

$$
\sum_{n=1}^{\infty}\left(s_{n}^{2 k}(C)-\left(\frac{4}{\lambda_{n}}\right)^{k}\right)=\operatorname{tr}\left(\left(C^{*} C\right)^{k}-\left(A^{*} A\right)^{k}\right) .
$$

В частности, при $k=1$ получаем

$$
\sum_{n=1}^{\infty}\left(s_{n}^{2}(C)-\frac{4}{\lambda_{n}}\right)=\operatorname{tr}\left(C^{*} C-A^{*} A\right)=\operatorname{tr} B^{*} B
$$

Так как

$$
\operatorname{tr} B^{*} B=\frac{1}{\pi^{2}} \int_{\Omega} \int_{\Omega}\left|\frac{1}{z-\xi}-\frac{F^{\prime}(z)}{F(z)-F(\xi)}-\frac{\overline{F(\xi)} F^{\prime}(z)}{1-\overline{F(\xi)} F(z)}\right|^{2} d A(\xi) d A(z)
$$

из предыдущего неравенства получаем (9).

\section{СПИСОК ЦИТИРОВАННОЙ ЛИТЕРАТУРЫ}

[1] Anderson J. M., Hinkkanen A. The Cauchy transform on bounded domain // Proc. Amer. Math. Soc. 1989. V. 107. P. 179-185.

[2] Гохберг И. Ц., Крейн М. Г. Введение в теорию линейных несамосопряженных операторов. М.: Наука, 1965.

[3] Watson G. N. A Treatise of the Theory of Bessel Functions. 2nd edition. Cambridge: Cambridge Univ. Press, 1962.

[4] Бейтман Г., Эрдейи А. Высшие трансцендентные функции. Т. 2. М.: Наука, 1974.

[5] Dostanić M.R. Norm estimate of the Cauchy transform on $L^{p}(\Omega) / /$ Integral Equations Operator Theory. (to appear).

[6] Boyd D. W. Best constants in a class of integral inequalities // Pacific J. Math. 1969. V. 30. № 2. P. 367-383.

[7] Dostanić M. R. The properties of the Cauchy transform on a bounded domain // J. Operator Theory. 1996. V. 36. P. 233-247.

[8] Векуа И.Н. Обобщенные аналитические функции. М.: Наука, 1988.

[9] Dostanić M. R. Spectral properties of the Cauchy operator and its product with Bergman's projection on a bounded domain // Proc. London Math. Soc. (3). 1998. V. 76. P. 667-684.

[10] Dostanić M. R. Spectral properties of the operator or Riesz potential type // Proc. Amer. Math. Soc. 1998. V. 126. № 8. P. 2291-2297.

[11] Горбачук В. И., Горбачук М. Л. Граничные задачи для дифференциальных уравнений с операторными коэффициентами. Киев: Наукова думка, 1984.

[12] Anderson J. M., Khavinson D., Lomonosov V. Spectral properties of some integral operators arising in potential theory // Quart. J. Math. (Oxford). Ser. 2. 1992. P. 387-407. 